\title{
Web-based nomograms for predicting the prognosis of adolescent and young adult skin melanoma, a large population-based real- world analysis
}

\author{
Chen Yang", Fei Liao", Li Cao \\ Department of Dermatology, The Affiliated Suzhou Science \& Technology Town Hospital of Nanjing Medical University, Suzhou, China \\ Contributions: (I) Conception and design: L Cao; (II) Administrative support: L Cao; (III) Provision of study materials or patients: C Yang, F Liao; (IV) \\ Collection and assembly of data: C Yang, F Liao; (V) Data analysis and interpretation: C Yang, F Liao; (VI) Manuscript writing: All authors; (VII) \\ Final approval of manuscript: All authors. \\ "These authors contributed equally to this work. \\ Correspondence to: Li Cao, MD. Department of Dermatology, The Affiliated Suzhou Science \& Technology Town Hospital of Nanjing Medical \\ University, Suzhou, China. Email: caoyy88@163.com.
}

Background: Invasive cutaneous melanoma is one of the most common malignant diseases among adolescents and young adults (aged 15-40 years) in the United States. We aimed to develop web-based nomograms to precisely predict overall survival and cancer-specific survival in this group of patients with cutaneous melanoma.

Methods: We analyzed the overall and caner-specific death events in 19,887 patients who underwent surgical resection of cutaneous melanoma from Surveillance, Epidemiology and End Results database and developed web-based clinic-pathologic prediction models for overall survival and cancer specific survival based on Cox regression. C-statistics of Harrell and time-dependent Receiver Operating Characteristic Curve (ROC) were used to evaluate the prognostic accuracy of nomograms.

Results: Multivariate Cox regression model analysis suggested that age, sex, race, tumor location, Clark level, ulceration, thickness, and $\mathrm{N}$ stage were independently associated with both overall survival and cancer-specific survival in adolescent and young adult patients with cutaneous melanoma. The nomograms performed excellently in predicting overall survival and cancer-specific survival with C-index being 0.875 (95\% CI: 0.847-0.903) and 0.901 (95\% CI: 0.876-0.925), respectively. Time-dependent ROC verified that the prognostic accuracy of nomograms was better than that of American Joint Committee on Cancer staging system and other prognostic factors.

Conclusions: These user-friendly nomograms can precisely predict overall survival and cancerspecific survival in cutaneous melanoma patients treated with surgical resection, which may help to make individualized postoperative follow-up and therapeutic schemes.

Keywords: Surveillance epidemiology and end results; melanoma; web-based nomogram

Submitted Mar 03, 2020. Accepted for publication Sep 12, 2020.

doi: $10.21037 /$ tcr-20-1295

View this article at: http://dx.doi.org/10.21037/tcr-20-1295 


\section{Introduction}

Cutaneous melanoma (CM) is one of the most common and lethal skin malignant disease among adolescents and young adults in the United States (1). The past decades have witnessed a constant increase of its incidence of in adolescents and young adults $(2,3)$. Compared with elder patients, these patients usually present different distribution patterns and possible distinct biological behaviors (4-7).

Though melanoma is characterized by the uncontrolled growth of pigment-producing cells, primary surgical resection offers the best opportunity of cure for patients with early-stage CM. However, even for CM patients treated with curative resection, long-term survival still varies significantly for patients with the same American Joint Committee on Cancer (AJCC) stage which is currently the most accepted prognosis predicting system and therapeutic decision making guideline (8). Therefore, many studies have been conducted to explore the potential prognostic factors associated with survival of CM to improve survival prediction (9-15). Nevertheless, limited previous studies focused on adolescent and young adult patients $(16,17)$ and no effective prognosis predicting model has been built to estimate long-term survival for these patients.

Therefore, the Surveillance Epidemiology and End Results (SEER) database was used to comprehensively evaluate the survival factors in adolescent and young adult CM patients treated with surgical resection. User-friendly web-based prediction nomograms were developed to estimate overall survival (OS) and cancer-specific survival (CSS) for adolescent and young adult CM patients to help to guide individualized therapeutic schemes and postoperative follow-up.

We have presented this article in accordance with the TRIPOD reporting checklist (available at http://dx.doi. org/10.21037/tcr-20-1295).

\section{Methods}

The study was conducted in accordance with the Declaration of Helsinki (as revised in 2013).

\section{Study population}

Data from the Surveillance Epidemiology and End Results (SEER) Program of the United States National Cancer Institute (released in 2016) were extracted and analyzed in this study. SEER database, collecting cancer incidence and survival data from 18 regional population registries, is a large population-based cancer registry. The SEER data are publicly available for studies of cancer-based epidemiology and clinical-pathological features of multiple kinds of cancers.

Inclusion criteria were as follows: (I) patients were aged 15-40 years old; (II) patients were pathologically confirmed as CM; (III) patients were diagnosed between year 2004 and 2014; (IV) all patients received surgical resection; (V) $\mathrm{CM}$ was the only primary tumor. Patients who met the following criteria were excluded in this study: (I) patients with distant metastatic site; (II) patients with unknown information of thickness or lymph node metastasis; (III) all patients were staged according to the AJCC Tumor-NodalMetastasis (TNM) Staging Classification for melanoma (Seventh Edition, 2010). Finally, a total of 19,887 patients were identified in our study.

\section{Statistical analysis}

The OS was defined as the time from treatment to death from any cause, and the CSS was defined as the time from treatment to the death from skin melanoma. Univariate analysis was used to examine the association between various prognostic predictors and OS, CSS. Significant prognostic predictors associated with OS and CSS were included to perform multivariate analyses by using the Cox proportional hazards model. $\mathrm{P}<0.05$ was considered statistically significant. As several clinicopathological variables except for $\mathrm{T}$ and $\mathrm{N}$ stage in the SEER database had missing data, and simply excluding patients with missing data is inefficient and would increase the risk of selection bias, we used multiple imputation method to reinput the missing data before analysis.

Web based nomograms were developed using $\mathrm{R}$ and JavaScript, and the model performance for prognostic accuracy was evaluated by time-dependent receiver operating characteristic curve (ROC) and Harrell's concordance index (c-index). All statistical evaluation was conducted with R 2.15.0 software (Institute for Statistics and Mathematics, Vienna, Austria).

\section{Results}

\section{Patients' baseline characteristics}

Among the 19,887 adolescent and young adult patients, $7,378(37.1 \%)$ are men and $12,509(62.9 \%)$ are women; 
8,384 cases are with superficial spreading melanoma, 1,036 with nodular melanoma, and 954 with other uncommon melanomas. As for the tumor Clark level, 8,171 (40.70\%) are level II; 5,600 (27.80\%) are level III, 6,116 (30.00\%) are level IV and 294 (1.50\%) are level IV. In terms of the AJCC TNM stage, stage I, stage IIA, stage IIB, stage IIC, and stage III have 16,991 (85.44\%), 837 (4.21\%) were, $433(2.18 \%), 123(0.62 \%)$ and $1,503(7.56 \%)$ patients respectively at the time of diagnosis. The detailed demographic and characteristics and imputation percentage are presented in Table 1 .

\section{Prognostic factors for OS and CSS}

To evaluate the association between baseline characteristics and prognosis, univariate Cox regression analysis were performed. In univariate analysis, age, sex, race, tumor location, histologic subtype, Clark level, ulceration, thickness, and $\mathrm{N}$ stage were significantly associated with OS and CSS $(\mathrm{P}<0.05)$. Multivariate Cox regression model was further conducted to evaluate the independence of the above significant prognostic factors. In both models of OS and CSS, age, sex, race, tumor location, Clark level, ulceration, thickness, and $\mathrm{N}$ stage were verified to be independent prognostic factors in patients with $\mathrm{CM}$ (Tables $2,3, \mathrm{P}<0.05)$. Histologic subtype lost its independence in predicting OS and CSS.

\section{Development and performance evaluation of prediction models}

To predict OS and CSS of skin melanoma patients, two nomograms were established by multivariate Cox regression model according to all significantly independent factors for OS and CSS (Figure 1A,B). Nomograms can be interpreted by summing up the points assigned to each variable, which is indicated at the top scale. The total points can be converted to predicted 5 -year probability of death and recurrence or metastasis for a patient in the lowest scale. The Harrell's c-indexes for OS and CSS prediction were 0.875 (95\% CI: $0.847-0.903)$ and 0.901 (95\% CI: $0.876-$ $0.925)$, respectively. Calibration curves for two nomograms (Figure 1C,D) revealed no deviations from the reference line and there is no need for recalibration.

Time dependent ROC analysis suggested that the nomograms we developed are more accurate in predicting OS and CSS than the AJCC stage and other prognostic factors (Figure 2). A histogram of nomogram-predicted probabilities within each of the AJCC stage is shown in Figure 3 and depicts the variation in predicted outcome within each of the AJCC version 3 subgroups.

To create user-friendly accessibility, the underlying statistical formulas were implemented in webbased nomograms. Patients or medical workers can estimate individual OS and CSS by entering the basic clinicopathological information, time of prediction. Figure 4 shows a screenshot of the web-based nomograms which are available on http://youthcm.site/.

\section{Discussion}

Although many studies have identified clinical and molecular predictors for long term survival after resection of $\mathrm{CM}$, limited researches focused on improving the evaluation of prognosis for adolescent and young adult patients and no practical and user-friendly predictive models have been developed. To our knowledge, we for the first time analyzed OS and CSS predicting factors comprehensively and built user-friendly web-based nomograms to precisely predict long term survival for adolescent and young adult patients based on SEER database. The web-based nomograms we constructed perform excellently in predicting OS and CSS with C-index being 0.875 (95\% CI: $0.847-0.903)$ and 0.900 (95\% CI: 0.876-0.925), respectively.

In this study, we found that age, sex, race, tumor location, Clark level, ulceration, thickness, and $\mathrm{N}$ stage are independently associated with OS and CSS. These variables have been reported in previous studies conducted in the overall CM patients (18-20). Consistent with published studies, older age, male, non-white, high Clark level, present ulceration, nodular melanoma, head and neck melanoma, and advanced tumor stage are related to elevated probability of melanoma mortality. Notably, univariate analysis suggested histological subtype is a prognostic factor and the nodular CM have worse prognosis than patients with superficial spreading melanoma. However, after adjusting other factors, histological subtype is not an independent OS and CSS predictor. Previous studies covering all age groups have confirmed that nodular melanoma is characterized with aggressive biological behavior and is an independent prognostic factor for poor survival $(21,22)$. The possible reason accounting for this discrepant result in our study can be firstly inferred that significant confounding bias caused by other prognostic factors exists among different histological subtype groups. Actually, further underlying reasons could attribute to the great heterogeneity among 
Table 1 Clinicopathological features of adolescent and young adult melanoma patients

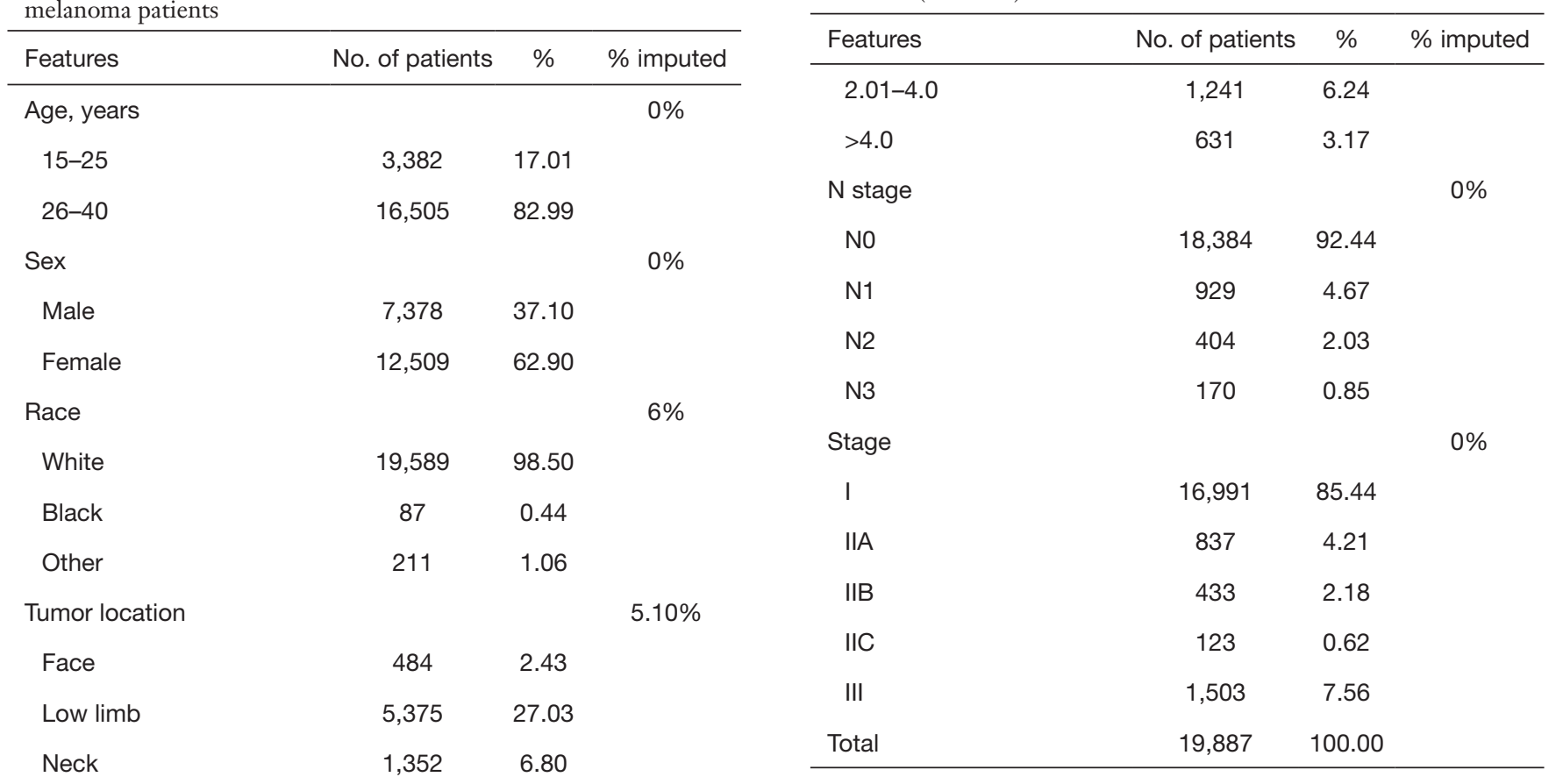

Trunk

Up limb

Overlapping

Histologic subtype

Superficial spreading

melanoma

Nodular melanoma

Other uncommon

melanomas

Unspecific

8,256

41.51

$4,404 \quad 22.15$

16

0.08

$0 \%$

$8,384 \quad 42.16$

$1,036 \quad 5.21$

$954 \quad 4.80$

$9,513 \quad 47.84$

Clark level

II

III

8,171

40.70

III

5,600

27.80

IV

6,116

30.00

$\mathrm{V}$

294

1.50

Ulceration

\section{No}

18,299

92.01

Yes

1,588

7.99

Thickness

$\leq 1$

15,232

76.59

1.01-2.0

2,783
$12 \%$
Table 1 (continued)

adolescent and young adult and the elderly patients (23). Consequently, the prognostic role of histological subtype should be re-evaluated comprehensively among CM patients at different ages and future well organized prospective studies are warranted to explore the inherent heterogeneity among different histological subtypes in adolescent and young adult patients.

The nomograms are a well-established prediction tool that incorporates significant clinicopathologic graphical representation of a statistical prediction model that generates a numerical probability of a clinical event. Although the current AJCC TNM staging system is the most influencing prognosis evaluating estimator for $\mathrm{CM}$ patients, many valuable prognostic variables, such as age, tumor location and Clark level are not incorporated. As revealed in our study, older age, male, non-white, high Clark level and neck melanoma are independent predictive factors of CM long-term survival. It is easy to imagine that distinct bias in estimating the prognosis cannot be avoided when we considered only TNM stage regardless of other important factors. As illustrated in figure 3 which is a histogram of predicted outcome based on the nomograms for each AJCC stage classification, significant heterogeneity in outcome can be found in each sub-stage, especially in factors known to impact survival. It is known as a simple

Table 1 (continued) 
Table 2 Univariate and multivariate analysis of overall survival (OS) in skin melanoma

\begin{tabular}{|c|c|c|c|c|}
\hline \multirow{2}{*}{ Features } & \multicolumn{2}{|c|}{ Univariate analysis } & \multicolumn{2}{|c|}{ Multivariate analysis } \\
\hline & $\mathrm{HR}(95 \% \mathrm{Cl})$ & $\mathrm{P}$ & $\mathrm{HR}(95 \% \mathrm{Cl})$ & $\mathrm{P}$ \\
\hline Age, years & & 0.015 & & $<0.001$ \\
\hline $26-40$ & $1.30(1.05-1.61)$ & & $1.64(1.32-2.04)$ & \\
\hline Sex & & $<0.001$ & & 0.001 \\
\hline Female & $0.44(0.38-0.51)$ & & $0.76(0.65-0.89)$ & \\
\hline Race & & $<0.001$ & & 0.002 \\
\hline White & 1 & & 1 & \\
\hline Black & $2.81(1.40-5.64)$ & & $1.13(0.55-2.31)$ & \\
\hline Other & $2.68(1.68-4.29)$ & & $2.19(1.36-3.53)$ & \\
\hline Low limb & $0.62(0.38-1.02)$ & & $0.72(0.44-1.16)$ & \\
\hline Neck & $1.45(0.86-2.43)$ & & $1.24(0.74-2.07)$ & \\
\hline Trunk & $1.029(0.64-0.65)$ & & $1.24(0.77-1.99)$ & \\
\hline Up limb & $0.83(0.51-1.36)$ & & $1.09(0.66-1.78)$ & \\
\hline Overlapping & $1.88(0.25-14.11)$ & & $4.89(0.64-37.01)$ & \\
\hline Histologic subtype & & $<0.001$ & & 0.194 \\
\hline Superficial spreading melanoma & 1 & & 1 & \\
\hline Nodular melanoma & $6.45(5.24-7.94)$ & & $0.88(0.69-1.11)$ & \\
\hline Other uncommon melanomas & $1.55(1.11-2.17)$ & & $0.78(0.56-1.11)$ & \\
\hline Ulceration & & $<0.001$ & & $<0.001$ \\
\hline No & 1 & & 1 & \\
\hline Yes & 10.59 (9.12-12.29) & & $2.55(2.13-3.06)$ & \\
\hline Thickness & & $<0.001$ & & $<0.001$ \\
\hline$\leq 1$ & 1 & & 1 & \\
\hline $1.01-2.0$ & $5.61(4.54-6.92)$ & & 3.09 (2.43-3.95) & \\
\hline $2.01-4.0$ & $14.24(11.55-17.54)$ & & $4.71(3.59-6.18)$ & \\
\hline$>4.0$ & 31.58 (25.58-38.98) & & $7.50(5.57-10.10)$ & \\
\hline $\mathrm{N}$ stage & & $<0.001$ & & $<0.001$ \\
\hline NO & 1 & & 1 & \\
\hline N1 & $8.69(7.18-10.52)$ & & $2.23(1.80-2.76)$ & \\
\hline N2 & $13.98(11.18-17.49)$ & & $3.12(2.43-4.01)$ & \\
\hline N3 & $32.00(24.90-41.12)$ & & $5.10(3.83-6.79)$ & \\
\hline
\end{tabular}


Table 3 Univariate and multivariate analysis of cancer specific survival (CSS) in skin melanoma

\begin{tabular}{|c|c|c|c|c|}
\hline \multirow{2}{*}{ Features } & \multicolumn{2}{|c|}{ Univariate analysis } & \multicolumn{2}{|c|}{ Multivariate analysis } \\
\hline & HR $(95 \% \mathrm{Cl})$ & $P$ & $\mathrm{HR}(95 \% \mathrm{Cl})$ & $\mathrm{P}$ \\
\hline Age, years & & 0.045 & & $<0.001$ \\
\hline $26-40$ & $1.26(1.01-1.60)$ & & $1.70(1.33-2.19)$ & \\
\hline Sex & & $<0.001$ & & 0.001 \\
\hline Race & & $<0.001$ & & 0.013 \\
\hline White & 1 & & 1 & \\
\hline Black & $3.40(1.69-6.84)$ & & $1.29(0.63-2.64)$ & \\
\hline Other & $2.70(1.61-4.50)$ & & $2.14(1.27-3.60)$ & \\
\hline Neck & $1.41(0.81-2.44)$ & & $1.19(0.68-2.07)$ & \\
\hline Trunk & $0.96(0.58-1.59)$ & & $1.09(0.65-1.82)$ & \\
\hline Up limb & $0.74(0.44-1.26)$ & & $0.96(0.56-1.63)$ & \\
\hline Overlapping & $0.001(0-1000+)$ & & $0.001(0-1000+)$ & \\
\hline Histologic subtype & & & & 0.376 \\
\hline Superficial spreading melanoma & 1 & & 1 & \\
\hline Nodular melanoma & $7.66(6.11-9.60)$ & & $0.95(0.72-1.20)$ & \\
\hline Other uncommon melanomas & $1.64(1.13-2.39)$ & & $0.73(0.49-1.07)$ & \\
\hline Unspecific & $1.31(1.07-1.59)$ & & $0.89(0.73-1.10)$ & \\
\hline No & 1 & & 1 & \\
\hline Yes & $12.74(10.83-15.00)$ & & $2.77(2.28-3.37)$ & \\
\hline Thickness & & $<0.001$ & & $<0.001$ \\
\hline$\leq 1$ & 1 & & 1 & \\
\hline $1.01-2.0$ & 7.37 (5.78-9.41) & & $3.54(2.68-4.68)$ & \\
\hline $2.01-4.0$ & $18.29(14.36-23.30)$ & & 4.87 (3.58-6.63) & \\
\hline$>4.0$ & $43.42(34.16-55.20)$ & & $8.04(5.77-11.20)$ & \\
\hline $\mathrm{N}$ stage & & $<0.001$ & & $<0.001$ \\
\hline No & 1 & & 1 & \\
\hline N1 & $9.96(8.09-12.26)$ & & $2.30(1.83-2.89)$ & \\
\hline N2 & $17.15(13.54-21.72)$ & & $3.43(2.64-4.46)$ & \\
\hline N3 & $40.00(30.79-51.95)$ & & $5.63(4.17-7.59)$ & \\
\hline
\end{tabular}


A

Points

Sex

Age

Race

Clark

Ulceration

Site

Thickness

Nstage

Total Points

1-year overall survival

3-year overall surviva

5-year overall survival
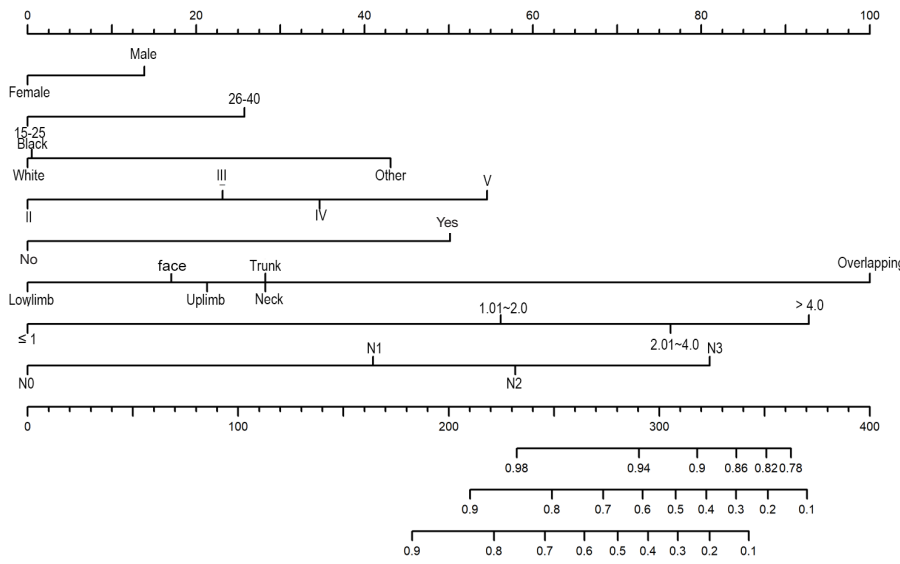

B

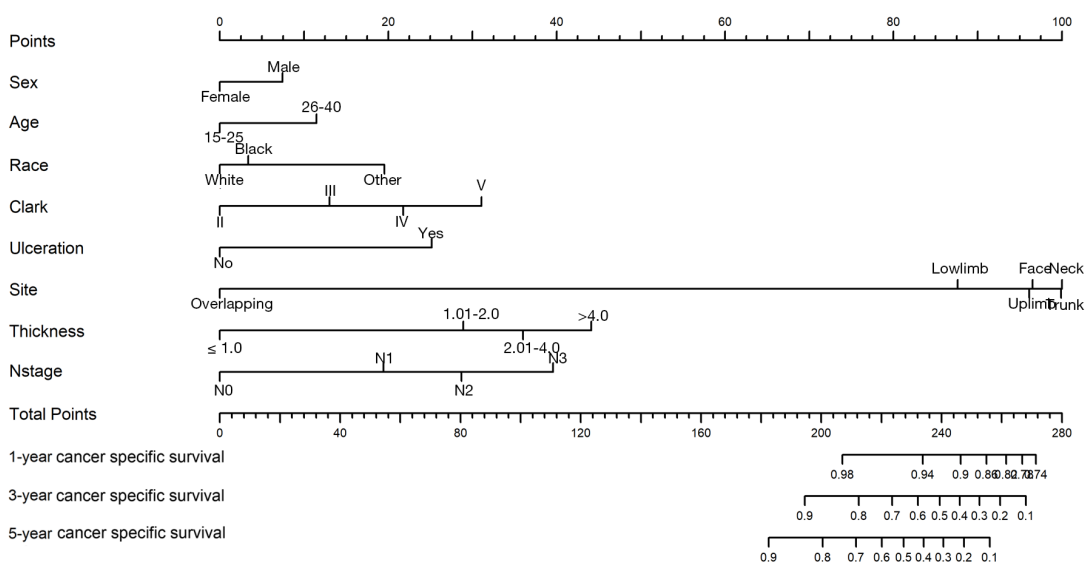

C

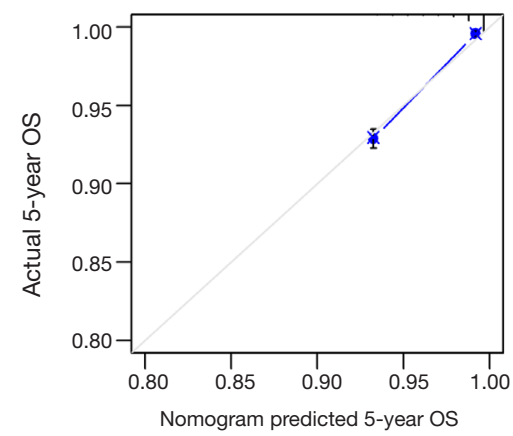

D

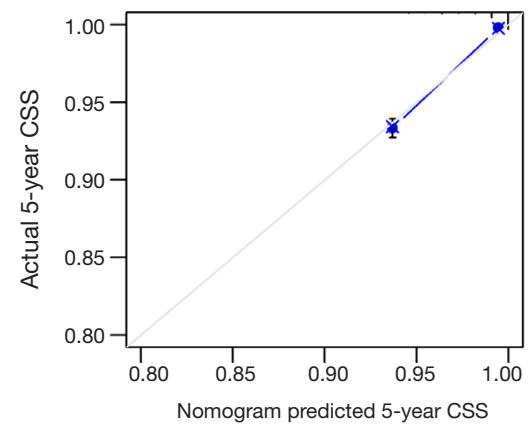

Figure 1 Nomograms with clinicopathological characteristics to predict OS (A) and CSS (B) of adolescent and young adult patients with CM. Calibration curves for 5-year OS (C) and 5-year CSS (D) using nomograms are shown. OS, overall survival; CSS, cancer specific survival; CM, cutaneous melanoma.

stage III. The nomograms developed in our study include not only AJCC staging system but also demographic characteristics. Furthermore, different from TNM staging system, the nomograms can provide quantified prognosis evaluation for individual patients. Though nomograms have been validated to compare favorably to the traditional TNM staging systems in many cancers, this graphical tool is still not convenient enough to be applied in clinical 

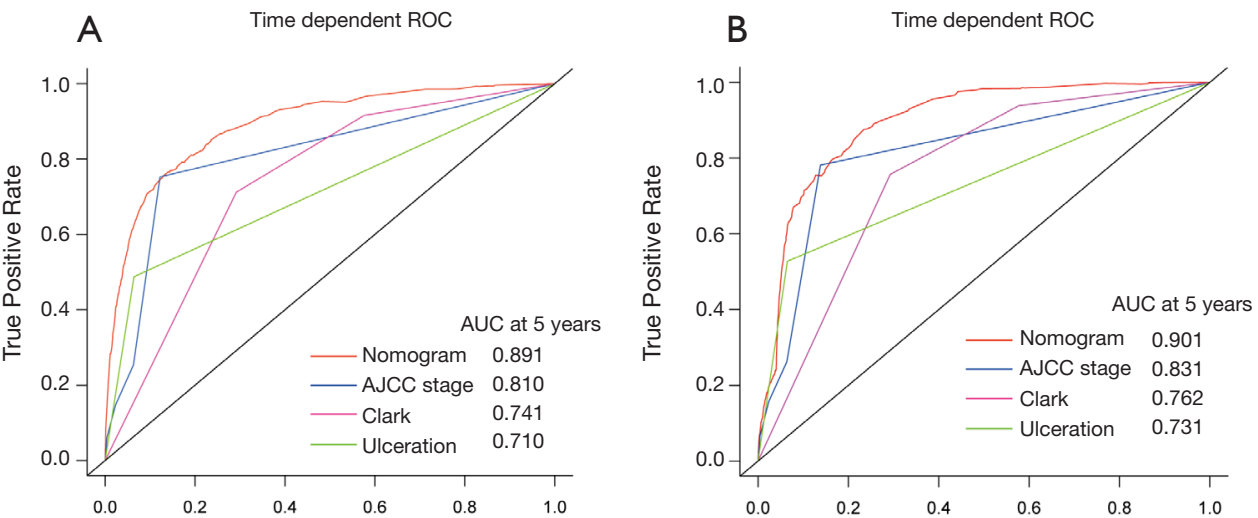

Figure 2 Time-dependent ROC curves at 5 year compare the prognostic accuracy in predicting OS (A) and CSS (B) of the nomograms with other clinicopathological features. OS, overall survival; CSS, cancer specific survival; ROC, receiver operating characteristic.
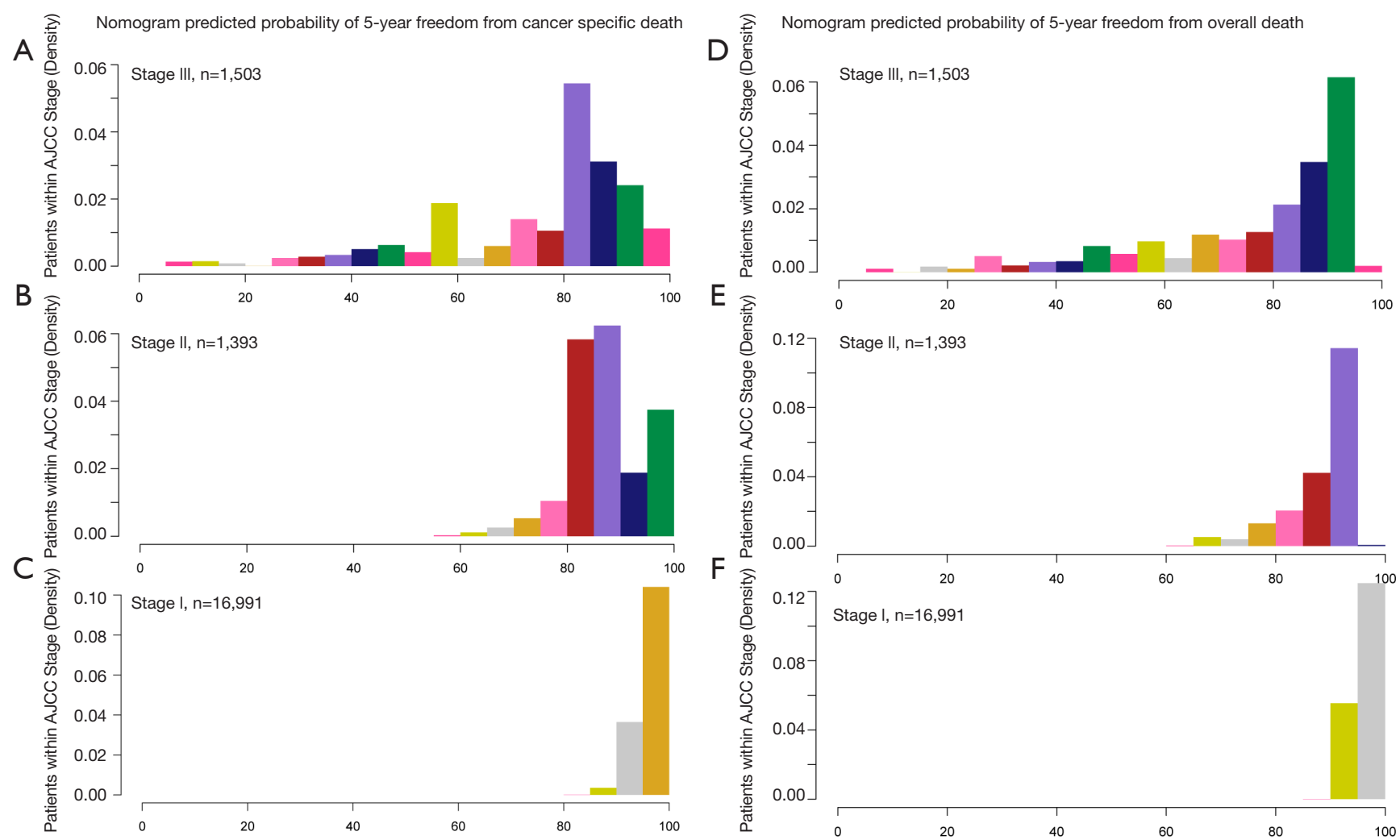

Figure 3 Histogram of nomogram-predicted freedom from cancer specific death (A,B,C) and overall death (D,E,F) within each AJCC stage. AJCC, American Joint Committee on Cancer.

practice. Therefore, we developed a user-friendly web-based nomogram to facilitate its feasibility in clinical practice. By typing in the clinicopathologic features of any individual patient, doctors can estimate the survival precisely.

Though the first web-based prognostic nomograms targeting adolescent and young adult patients has been built, there are still several limitations in our study. Firstly, SEER database lacks several important clinical information including tumor grade, comorbidity and disease-free survival. Secondly, this study only included young patients 


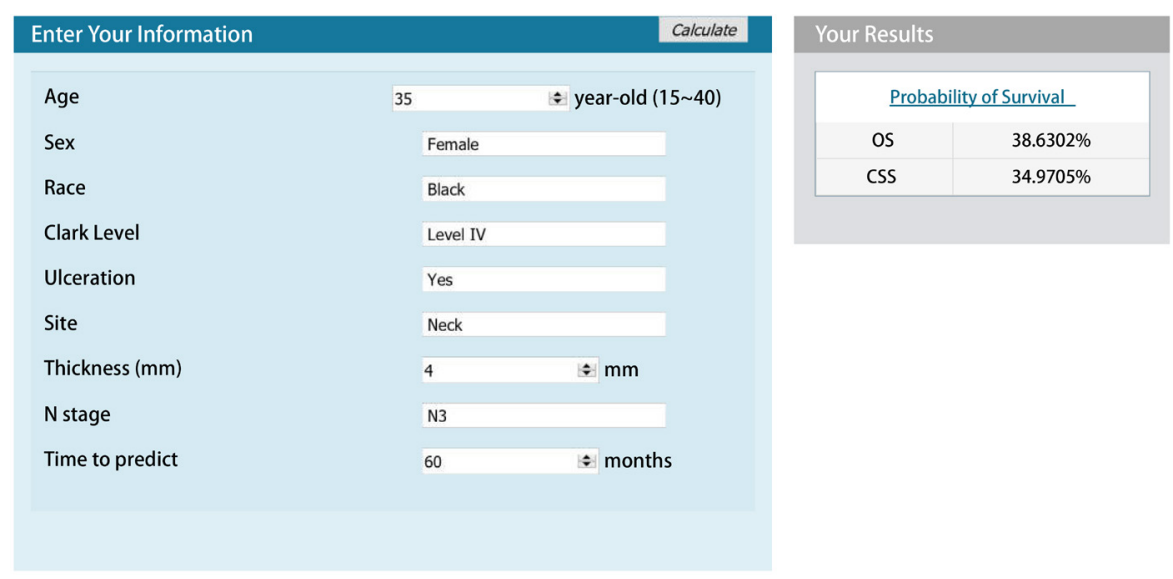

Figure 4 Print screen from the web-based nomograms, predicting OS and CSS in a fictional patient. The nomograms are available at http:// youthcm.site/. Choose or enter the value for each variable, and then press the "Calculate" button. OS, overall survival; CSS, cancer specific survival.

receiving surgical resection and thus this model cannot be applied to the elderly or patients with distant metastasis. Thirdly, external validation is needed to verify whether our predictive model is universally applicable.

\section{Conclusions}

In conclusion, we have developed precise user-friendly webbased nomograms to predict OS and CSS in adolescent and young adult CM patients based on a large population cohort. It is hoped that this personalized predictive tool can be applied to treatment and follow-up related decision making.

\section{Acknowledgments}

We thank the SEER database for providing these valuable data.

Funding: This study was supported by the program of New District Medical health science and Technology (No. 2018Q009).

\section{Footnote}

Reporting Checklist: The authors have completed the TRIPOD reporting checklist. Available at http://dx.doi. org/10.21037/tcr-20-1295

Conflicts of Interest: All authors have completed the ICMJE uniform disclosure form (available at http://dx.doi. org/10.21037/tcr-20-1295). The authors have no conflicts of interest to declare.

Ethical Statement: The authors are accountable for all aspects of the work in ensuring that questions related to the accuracy or integrity of any part of the work are appropriately investigated and resolved. The study was conducted in accordance with the Declaration of Helsinki (as revised in 2013).

Open Access Statement: This is an Open Access article distributed in accordance with the Creative Commons Attribution-NonCommercial-NoDerivs 4.0 International License (CC BY-NC-ND 4.0), which permits the noncommercial replication and distribution of the article with the strict proviso that no changes or edits are made and the original work is properly cited (including links to both the formal publication through the relevant DOI and the license). See: https://creativecommons.org/licenses/by-nc-nd/4.0/.

\section{References}

1. Bleyer A, Viny A, Barr R. Cancer in 15- to 29-year-olds by primary site. Oncologist 2006;11:590-601.

2. Senerchia AA, Ribeiro KB, Rodriguez-Galindo C. Trends in incidence of primary cutaneous malignancies in children, adolescents, and young adults: a populationbased study. Pediatr Blood Cancer 2014;61:211-6. 
3. Weir HK, Marrett LD, Cokkinides V, et al. Melanoma in adolescents and young adults (ages 15-39 years): United States, 1999-2006. J Am Acad Dermatol 2011;65:S38-49.

4. Lambie M, Nadler C, Glat P, et al. Infantile melanoma--a triple threat: diagnosis and management. Ann Plast Surg 2011;67:85-9.

5. Singh R, Shirali R, Chatterjee S, et al. Epidemiology of cancers among adolescents and young adults from a tertiary cancer center in Delhi. Indian J Med Paediatr Oncol 2016;37:90-4.

6. Bataille V, Winnett A, Sasieni P, et al. Exposure to the sun and sunbeds and the risk of cutaneous melanoma in the UK: a case-control study. Eur J Cancer 2004;40:429-35.

7. Ecsedi S, Hernandez-Vargas H, Lima SC, et al. DNA methylation characteristics of primary melanomas with distinct biological behaviour. PLoS One 2014;9:e96612.

8. Edge SB, Byrd DR, Compton CC, Fritz AG, Greene FL, Trotti III A. AJCC Cancer Staging Manual, 7th ed. Springer-Verlag: New York, 2010.

9. Balch CM, Gershenwald JE, Soong SJ, et al. Multivariate analysis of prognostic factors among 2,313 patients with stage III melanoma: comparison of nodal micrometastases versus macrometastases. J Clin Oncol 2010;28:2452-9.

10. Spillane AJ, Cheung BL, Winstanley J, et al. Lymph node ratio provides prognostic information in addition to american joint committee on cancer $\mathrm{N}$ stage in patients with melanoma, even if quality of surgery is standardized. Ann Surg 2011;253:109-15.

11. Chen J, Xu Y, Zhou Y, et al. Prognostic role of sentinel lymph node biopsy for patients with cutaneous melanoma: A retrospective study of surveillance, epidemiology, and end-result population-based data. Oncotarget 2016;7:45671-7.

12. Wasif N, Etzioni D, Haddad D, et al. Staging studies for cutaneous melanoma in the United States: a populationbased analysis. Ann Surg Oncol 2015;22:1366-70.

13. Abdel-Rahman O. Clinical correlates and prognostic value of different metastatic sites in patients with malignant melanoma of the skin: a SEER database analysis. J Dermatolog Treat 2018;29:176-81.

Cite this article as: Yang C, Liao F, Cao L. Web-based nomograms for predicting the prognosis of adolescent and young adult skin melanoma, a large population-based realworld analysis. Transl Cancer Res 2020;9(11):7103-7112. doi: $10.21037 /$ tcr-20-1295
14. Bagaria SP, Ray PS, Joseph RW, et al. Ultrathin primary is a marker for worse prognosis in lymph node-positive cutaneous melanoma. Cancer 2013;119:1860-7.

15. Mocellin S, Pasquali S, Rossi CR, et al. Validation of the prognostic value of lymph node ratio in patients with cutaneous melanoma: a population-based study of 8,177 cases. Surgery 2011;150:83-90.

16. Sanchez PC, Noda AY, Franco DD, et al. Melanoma in children, adolescents, and young adults: a clinical pathological study in a Brazilian population. Am J Dermatopathol 2014;36:620-8.

17. Iannacone MR, Youlden DR, Baade PD, et al. Melanoma incidence trends and survival in adolescents and young adults in Queensland, Australia. Int J Cancer 2015;136:603-9.

18. Rees MJ, Liao H, Spillane J, et al. Localized melanoma in older patients, the impact of increasing age and comorbid medical conditions. Eur J Surg Oncol 2016;42:1359-66.

19. Shen W, Sakamoto N, Yang L. Melanoma-specific mortality and competing mortality in patients with nonmetastatic malignant melanoma: a population-based analysis. BMC Cancer 2016;16:413.

20. Marashi-Pour S, Morrell S, Cooke-Yarborough C, et al. Competing risk analysis of mortality from invasive cutaneous melanoma in New South Wales: a populationbased study, 1988-2007. Aust N Z J Public Health 2012;36:441-5.

21. Faut M, Wevers KP, van Ginkel RJ, et al. Nodular Histologic Subtype and Ulceration are Tumor Factors Associated with High Risk of Recurrence in Sentinel Node-Negative Melanoma Patients. Ann Surg Oncol 2017;24:142-9.

22. Carrera C, Gual A, Diaz A, et al. Prognostic role of the histological subtype of melanoma on the hands and feet in Caucasians. Melanoma Res 2017;27:315-20.

23. Lu X, Zhang Q, Wang Y, et al. Molecular classification and subtype-specific characterization of skin cutaneous melanoma by aggregating multiple genomic platform data. J Cancer Res Clin Oncol 2018;144:1635-47. 